we found that $9.6 \%$ of 114 consecutive asymptomatic women tested positive for severe acute respiratory coronavirus virus 2 (SARS-CoV-2). None of those 11 SARS-CoV-2-positive pregnant women had any COVID-19-related symptoms.

The much higher rate of asymptomatic COVID-19 infections that we found (9.6\% vs $1.5 \%$ ) cannot be explained by the 3 theories proposed by Goldfarb et al. Our Philadelphia COVID-19 testing data are from the same period as the Boston study, when physical distancing orders were also in place. Although Boston does have fewer people per square mile $(13,841)$ than New York City $(27,000)$, Philadelphia has an even lower population density (11,854 people per square mile). On March 28, 2020, prior to the Boston study's time frame (and ours), Governor Andrew Cuomo announced an executive order that New York hospitals were required to allow 1 person to accompany a patient throughout their labor and delivery. This was issued several days after 2 major New York City hospital systems banned support people from labor and delivery rooms because of the coronavirus pandemic in effort to protect patients, babies, and labor and delivery healthcare providers. $^{2}$

Based upon our findings, as well as others ${ }^{3}$, the very low rate of asymptomatic pregnant women infected with SARS-CoV-2 in the Boston study may be an outlier during the early stages of the pandemic, with a more accurate infection rate being much higher. The higher rate of asymptomatic pregnant women infected with SARSCoV-2, who may still infect healthcare providers and others, demonstrates the importance of universal testing of pregnant women admitted to labor and delivery, as well as precautions such as mask wearing and hand washing.

Acknowledgments. None.

Financial support. No financial support was provided relevant to this article.

Conflicts of interest. All authors report no conflicts of interest relevant to this article.

\section{References}

1. Goldfarb IT, Diouf K, Barth WH, et al. Universal SARS-CoV-2 testing on admission to the labor and delivery unit: low prevalence among asymptomatic obstetric patients. Infect Control Hosp Epidemiol 2020 May 27 [Epub ahead of print]. doi: 10.1017/ice.2020.255

2. Sutton D, Fuchs K, D'Alton M, Goffman D. Universal screening for SARSCoV-2 in women admitted for delivery. N Engl J Med 2020 Apr 13 [Epub ahead of print]. doi: 10.1056/NEJMc2009316.

3. Vintzileos WS, Muscat J, Hoffmann E, et al. Screening all pregnant women admitted to labor and delivery for the virus responsible for COVID-19. Am J Obstet Gynecol 2020 Apr 26 [Epub ahead of print]. doi: 10.1016/j.ajog.2020. 04.024.

\title{
Validation of a small-size pooling approach targeting hospital surveillance of SARS-CoV-2 infection
}

\author{
Andrea Petrucca $\mathrm{PhD}^{1}$ (1), Marina Borro $\mathrm{PhD}^{2,3}$, Luana Lionetto $\mathrm{PhD}^{2,3}$, Giovanna Gentile $\mathrm{PhD}^{2,3}$, Antonella Alari Biol ${ }^{1}$, \\ Maurizio Simmaco MD, $\mathrm{PhD}^{2,3}$ and Iolanda Santino MD, $\mathrm{PhD}^{1,2}$ \\ ${ }^{1}$ Microbiology Unit, Sant'Andrea Hospital, Rome, Italy, ${ }^{2}$ Department of Neurosciences, Mental Health and Sensory Organs, Sapienza University of Rome, Rome, \\ Italy and ${ }^{3}$ Sant'Andrea Hospital, Rome, Italy
}

To the Editor-The ongoing coronavirus disease 2019 (COVID-19) pandemic, caused by severe acute respiratory syndrome coronavirus 2 (SARS-CoV-2), started in December 2019 as a large viral pneumonia outbreak in the city of Wuhan, China. ${ }^{1}$ The disease spread from Wuhan to other countries, and the World Health Organization declared it a pandemic by March 11, 2020 (https://www.who. int/emergencies/diseases/novel-coronavirus-2019/events-as-theyhappen). With vaccine development currently underway, the rapid identification of disease carriers and their close contacts represents the only effective measure to limit SARS-CoV-2 spreading. ${ }^{2}$

Hospitals are hotbeds for SARS-CoV-2 transmission; healthcare workers (HCWs) are at high risk of being infected and of further transmitting the virus to vulnerable patients. ${ }^{3}$ Thus, infection control strategies based on SARS-CoV-2 testing in HCWs and patients are necessary. ${ }^{4}$ Unfortunately, this type of disease

Author for correspondence: Andrea Petrucca, E-mail: apetrucca@ospedalesantandrea.it Cite this article: Petrucca A, et al. (2021). Validation of a small-size pooling approach targeting hospital surveillance of SARS-CoV-2 infection. Infection Control \& Hospital Epidemiology, 42: 909-911, https://doi.org/10.1017/ice.2020.380 surveillance is limited by the overwhelming demand for SARSCoV-2 molecular diagnostic analyses. $3,5,6$

To increase COVID-19 testing capacity, procedures based on pooling of naso-oral pharyngeal (NOP) swab specimens have been recently proposed. ${ }^{7-9}$ However, the validation of the sample pooling approach is crucial to assess its diagnostic accuracy and to avoid false-negative results. Recent studies describing the detection of SARS-CoV-2 RNA in pools of 5 to 32 samples reported falsenegative rates up to $10 \%$ for large groups, suggesting that smaller sample pools are a good compromise to increase sample processing capacity while maintaining test reliability. ${ }^{6-9}$ Since 5 -sample pools were shown to efficiently detect SARS-CoV-2 RNA in RT-PCR assays, ${ }^{7}$ we chose to test and validate this approach using a highthroughput RNA extraction and amplification platform. The Sant' Andrea Hospital of Rome (Italy) has put in place a SARSCoV-2 surveillance program focused on the periodic screening of HCWs and preventive screening of patients (before hospitalization). In total, 2,035 people from the surveillance program $(1,437 \mathrm{HCWs}$ and 598 patients) were enrolled in this study. The molecular diagnostic workflow we used for SARS-CoV-2 detection included the following elements: (1) NOP swab sampling using the COPAN UTM-RM virus transport medium (Copan Diagnostics,

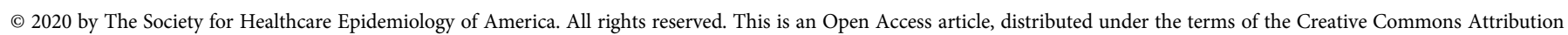

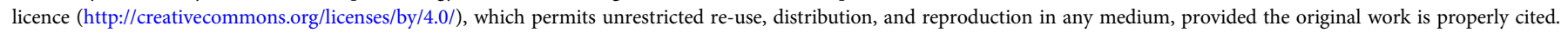


Fig. 1. Influence of pooling samples strategy on the sensitivity of RT-PCR. Cycle threshold $(\mathrm{Ct})$ values obtained from individual positive naso-oral pharyngeal swabs (NOP) samples (P1-P10; black symbols) and from their corresponding pooled samples, run in duplicate (open and grey symbols). Circles and squares indicate $\mathrm{Ct}$ values of the $\mathrm{N}$ and Orf1ab genes, respectively. Connecting brackets indicate the change in $\mathrm{Ct}(\Delta \mathrm{Ct})$ between individual NOP positive samples and their corresponding pools. The horizontal dotted line represents the $\mathrm{Ct}$ limit of our RT-PCR assay to assign a positive detection of SARS-Cov-2 RNA in NOP specimens.

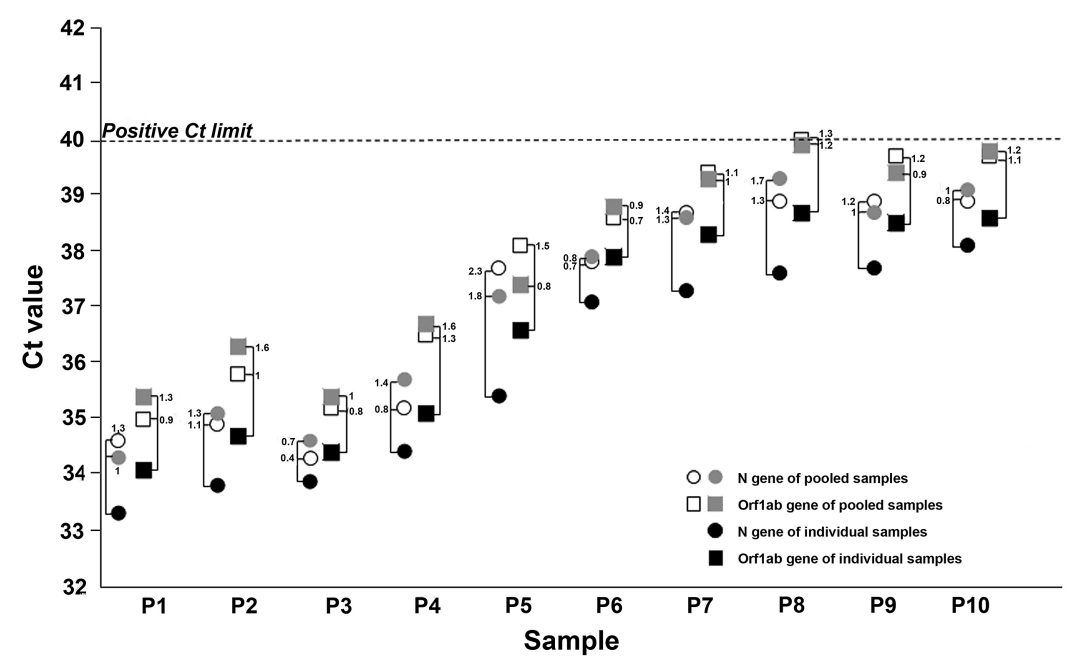

Murrieta, CA); (2) automated specimen RNA extraction and amplification with the Versant kPCR molecular system (Siemens Healthineers AG, Erlangen, Germany). Viral nucleic acid detection was carried out using the detection kit for 2019 novel coronavirus (2019-nCoV) RNA (PCR-fluorescence probing; Daan Gene, Sun Yat University, Guangzhou, Guandong, China), an RT-PCR assay which simultaneously detects the viral nucleocapsid $(\mathrm{N})$ and Orflab genes.

We first tested a small set of NOP swab pools to assess the lower detection limit of the method, then we validated the method on a larger set of sample pools from Sant' Andrea Hospital HCWs and patients. Each sample was analyzed both individually and as a part of a pool of 5 specimens $(200 \mu \mathrm{L}$ each). The small set consisted of 10 pools, each including 1 SARS-CoV-2-positive sample and 4 negative NOP samples. For each pool, 2 technical replicates were prepared and analyzed. The PCR cycle threshold $(\mathrm{Ct})$ values of individually tested positive samples ranged from 33.3 to 38.1 for the $\mathrm{N}$ gene and from 34.1 to 38.7 for the Orflab gene, whereas $\mathrm{Ct}$ values obtained from their corresponding pools were between 34.3 and 38.9 for the $\mathrm{N}$ gene and between 35 and 40 for the Orflab gene (Fig. 1). The $\mathrm{Ct}$ value differences $(\Delta \mathrm{Ct})$ between individual and pooled positive samples ranged from 0.3 to 2.3 for $\mathrm{N}$ and from 0.4 to 1.8 for Orflab (Fig. 1). No false-positive amplification signals were obtained using an analogous set of sample pools consisting of only SARS-CoV-2-negative NOP specimens.

We next performed a validation of the pooling strategy to assess the diagnostic performance and benefits of this approach. Daily during the first 3 weeks of April 2020, we analyzed an average of 96.9 individual NOP samples and their corresponding 19.38 pools collected from Sant' Andrea Hospital HCWs and patients (2,035 individual samples and 407 pools). In total, 36 patients (1.7 \%) were identified as SARS-CoV-2-positive through the analysis of individual samples as well as of their corresponding pools. Interestingly, all SARS-CoV-2-positive study participants belonged to the HCW group. In individually tested positive NOP specimens, the average Ct value for the $\mathrm{N}$ gene was $29.6( \pm 4.7)$ and the average $\mathrm{Ct}$ value for the Orflab gene was $31.1( \pm 5.6)$. In pooled samples, the average $\mathrm{Ct}$ value for the $\mathrm{N}$ gene was $31.7( \pm 5.9)$ and the average Ct value for the Orflab gene was $33.8( \pm 6.1)$.

The diagnostic accuracy of the 5-sample pooling strategy was excellent, showing sensitivity, specificity, and positive and negative predictive values of $100 \%$. The tests required to complete individual NOP sample and pool analysis were, respectively, 2,035 and 587 (407 pools plus $36 \times 5=180$ tests to confirm single samples included in positive pools). Summarizing, the small-pooling approach saved 1,448 tests, corresponding to $71.1 \%$ of the total cost of laboratory reagents required for individual sample analysis (ie, 15 RNA extraction and RT-PCR amplification kits). In our hands, it was possible to run at least 2 consecutive analytical sessions per day, allowing the reanalysis of individual samples from positive pools within 24 hours, which is a standard laboratory turnaround time for SARS-CoV-2 diagnostics in Italy.

When COVID-19 incidence is low, as in our study (below 2\%), the small-pooling approach significantly reduces the use of laboratory resources and simultaneously increases the number of screened people. The number of positive pools to be reanalyzed increases in relation to SARS-CoV-2 incidence, consequently worsening TAT and cost-benefit ratio. In conclusion, the described approach represents an optimal strategy for surveillance programs in late pandemic phases when screening of a large population is needed.

\section{Acknowledgments. None.}

Financial support. No financial support was provided relevant to this article.

Conflicts of interest. All authors report no conflicts of interest relevant to this article.

\section{References}

1. Zhu N, Zhang D, Wang W, et al. Novel coronavirus from patients with pneumonia in China, 2019. N Engl J Med 2020;382:727-733.

2. Munster VJ, Koopmans M, van Doremalen N, van Riel D, de Wit E. A novel Coronavirus emerging in China—key questions for impact assessment. N Engl J Med 2020;382:692-694.

3. Keeley AJ, Evans C, Colton $\mathrm{H}$, et al. Roll-out of SARS-CoV-2 testing for healthcare workers at a large NHS Foundation Trust in the United Kingdom. Euro Surveill 2020;25:2000433.

4. Livingston E, Bucher K. Coronavirus disease 2019 (COVID-19) in Italy. JAMA 2020;323:1335. doi: 10.1001/jama.2020.4344.

5. Syril DP, Keith RJ, Rouquié D, et al. 'All in': a pragmatic framework for COVID-19 testing and action on a global scale. EMBO Mol Med 2020: e12634. 
6. Cheng MP, Papenburg J, Desjardins M, et al. Diagnostic testing for severe acute respiratory syndrome-related coronavirus 2: Ann Intern Med 2020; 172:726-734.

7. Abdalhamid B, Bilder CR, McCutchen LE, Hinrichs SH, Koepsell SA, Iwen PC. Assessment of specimen pooling to conserve SARS CoV-2 testing resources. Am J Clin Pathol 2020;153:715-718.
8. Yelin I, Aharony N, Shaer Tamar E, et al. Evaluation of COVID-19 RT-qPCR test in multisample pools. Clin Infect Dis 2020:ciaa531.

9. Lohse S, Pfuhl T, Berkó-Göttel B, et al. Pooling of samples for testing for SARS-CoV-2 in asymptomatic people. Lancet Infect Dis 2020 Apr 28 [Epub ahead of print]. doi: 10.1016/S1473-3099(20)30362-5.

\title{
Configuring a hospital in the COVID-19 era by integrating crisis management logistics
}

\author{
Abdulrahman Alharthy $\mathrm{MD}^{1}$, Fahad Faqihi MD${ }^{1}$, Huda Mhawish $\mathrm{MSc}^{1}$, Abdullah Balhamar $\mathrm{MD}^{1}$, \\ Ziad A. Memish MD, FACP ${ }^{2}$ and Dimitrios Karakitsos MD, PhD $^{1}$ (1) \\ ${ }^{1}$ Critical Care Department, King Saud Medical City, Riyadh, Kingdom of Saudi Arabia and ${ }^{2}$ Research \& Innovation Centre, King Saud Medical City, Riyadh, \\ Kingdom of Saudi Arabia
}

To the Editor-The novel coronavirus SARS-CoV-2 disease (COVID-19) emerged in China and has spread throughout the world. ${ }^{1}$ The first case of COVID-19 in Saudi Arabia was confirmed on March 2, 2020, and presently almost 200,000 people have been infected here. ${ }^{2}$ The Ministry of Health (MOH) has responded to the COVID-19 outbreak by designing clusters of governmental hospitals to accommodate the increased flow of patients. Although our bed-occupancy rates never exceeded $80 \%$ until 2019, the situation has changed dramatically since March 2020, when the intensive care unit (ICU) occupancy rates reached $100 \%$ due to the pandemic. Hence, our hospital has been under pressure to upgrade our ICU services. We have used crisis management tactics in configuring our medical city (Table 1). First, we created a multidisciplinary crisis management team (CMT) to supervise the operations, and we promptly applied a surge plan based on the available scientific evidence. Our CMT policies, ICU configuration strategy, staff and resource utilization, admission protocols, and therapeutic guidelines have been reviewed continually based on new international updates, emerging therapies, and the recommendations of our national health authorities. $^{2-5}$ By adjusting, and retrofitting existing ICUs, and acute wards. we have expanded the ICU bed capacity in a stepwise manner: phase 1, 180 beds; phase 2, 240 beds; phase 3, 300 beds). Our main challenge has been to install new structures (ie, gas access, power circuits, monitors, and HEPA purifiers) in the pop-up units. We could not maintain single-patient occupancy; thus, we isolated cohorts of COVID-19 patients in multiple-occupancy glass rooms. Nursing stations have been set up outside these rooms; new circuits have been installed for the transmission of data and alarms; and new procedure carts have been arranged for each new unit. The ICU-bed triage and

Author for correspondence: Dr Dimitrios Karakitsos, E-mail: karakitsosdimitrios@ gmail.com

Cite this article: Alharthy A, et al. (2021). Configuring a hospital in the COVID-19 era by integrating crisis management logistics. Infection Control \& Hospital Epidemiology, 42: 911-913, https://doi.org/10.1017/ice.2020.365 staff governance have been controlled by the CMT. Our CMT members have provided coverage 24 hours per day, 7 days per week to arrange the ICU admission flow and the transfer of patients to other hospitals based on the daily $\mathrm{MOH}$ plan. We have followed a tiered strategy in which we allocate experienced intensivists and nurses to supervise redeployed noncritical care physicians and nurses, and we also established back-up teams. The refinement of the respiratory and ICU care included changing the ventilator circuits and filters based on patient needs, avoiding nebulizers, creating specialized intubation and prone-positioning ventilation teams, and upgrading the oxygen supply system. The latter has been a major problem for our oxygen supply management team. Hence, we have promoted awake prone positioning and more oxygen-support therapies (ie, high-flow nasal cannula, and helmet continuous positive pressure ventilation) to avoid mechanical ventilation if possible. Interventional therapies (ie, extracorporeal membrane oxygenation and therapeutic plasma exchange) have been carefully screened by expert teams to optimize resource utilization. The ICU pharmacy operations have been linked to the $\mathrm{MOH}$ central stock and supervised by pharmacists of the CMT to facilitate the prompt delivery of medications. Infection control measures have been strictly implemented in all hospital areas by creating specific zones and protocols for donning and doffing personal protective equipment, providing sanitizer dispensers, applying strict room-disinfection protocols, and providing safe waste handling. ${ }^{6}$ Moreover, we have utilized novel transportation capsule isolation technology to minimize the risk of SARS-CoV-2 acquisition during inter- and intrahospital transportation. ${ }^{?}$

New hospital communication systems have been installed in the pop-up ICUs. The communication between frontline staff and the CMT is continuous. Because visitors were not allowed, family meetings were organized via web-based applications to reduce patient and family stress. Training and emotional staff support have been provided on a daily basis. We have utilized daily COVID-19 training sessions to provide additional emotional reassurance (ie, dual training and emotional support strategy). Moreover, 600 COVID-19 patients have been hospitalized in our ICU over the past 4 months. Our staff s nosocomial infection rate was $\sim 3 \%$ during the early stages of the pandemic, and it has decreased to $0.5 \%$ since May 2020. As the

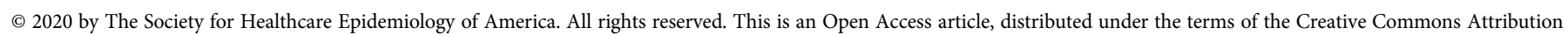

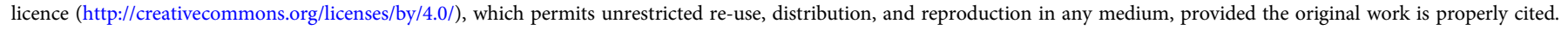

